\title{
Young doctors warned that social media vitriol could harm their mental health
}

\author{
n Cite as: CMAJ 2018 November 26;190:E1396-7. doi: 10.1503/cmaj.109-5677
}

Posted on cmajnews.com on Nov. 6, 2018.

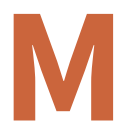

illennial doctors should limit social media time to protect their mental health, according to Dr. Clare Gerada, who made the statement ahead of the recent Wounded Healer conference in London, England. It was a dire warning from a physician well acquainted with burnout: Gerada heads the Practitioner Health Programme for the National Health Service, which provides support to stressed-out physicians in the United Kingdom.

Gerada advised young doctors to take themselves off social media for periods of time as a break from the online vitriol spewed by angry patients and lobby groups. Many Canadian medical professionals, however, suggest social media's pros outweigh the cons - as long as medical students and residents treat it as a tool in their ongoing education and are prepared to think before they tweet.

Two young doctors tackled this ongoing dialogue last year in an article called "Are medical trainees and doctors prepared for the new social media frontier?" Dr. Helene Retrouvey and Dr. Annie Wang, both plastic surgery residents, asked several physicians to comment on the role of social media in their profession.

"We noticed that more trainees are using social media for professional pur-

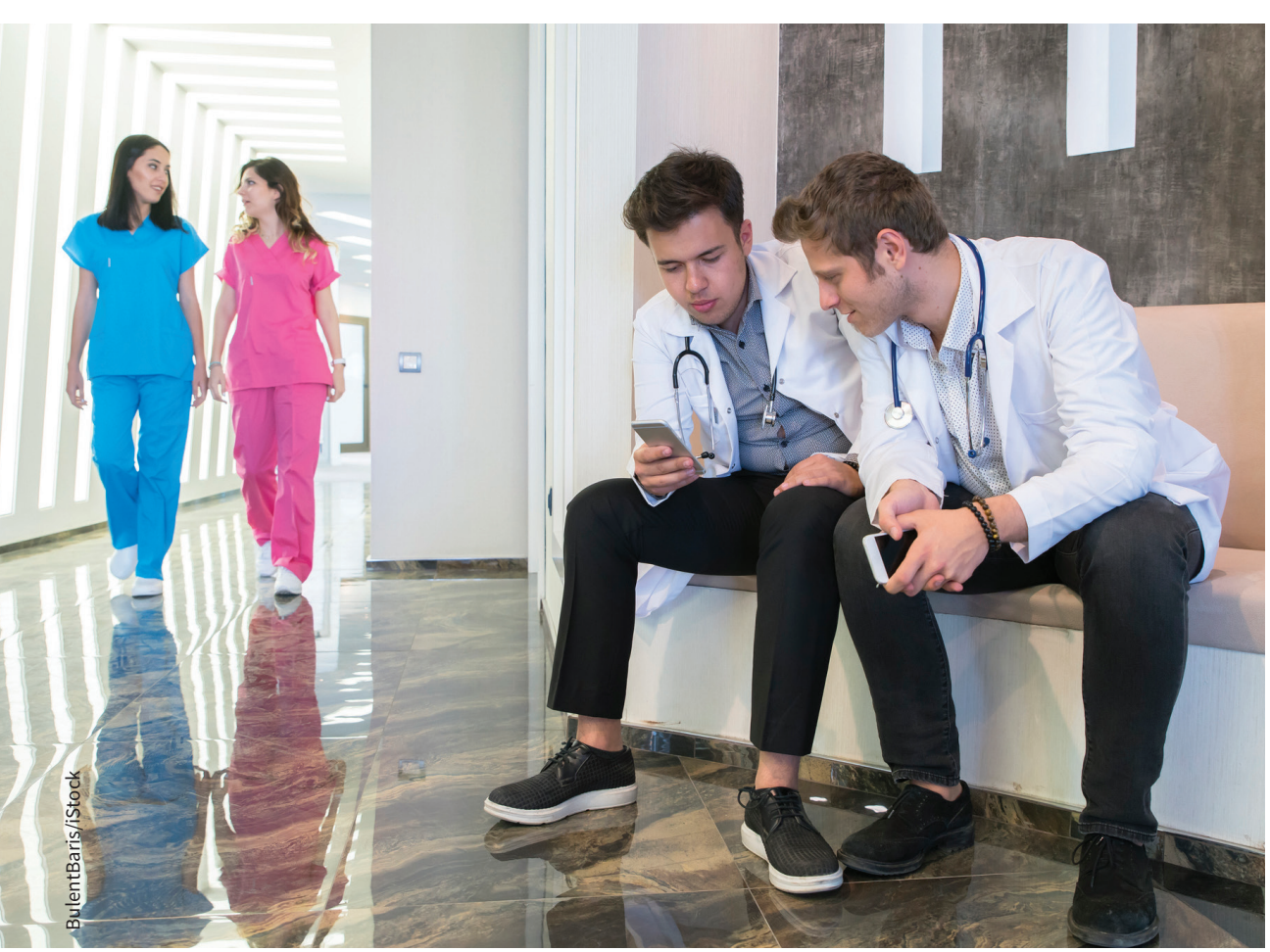

Whether social media has a positive or negative effect on young physicians depends on how they use it. poses. We wondered if social media was causing a blurring between professional and personal lives, and if trainees were prepared for this new reality," says Retrouvey. Some trainees shared stories of being exposed to online abuse and intimidation from colleagues. There was also concern that credibility is often measured by number of followers, not clinical experience.

The discussion's takeaway? Mastering social media is an ongoing process because it's still relatively new and evolving as a communication tool. With that in mind, Retrouvey says she and Wang believe medical trainees should receive more formal training not only on inappropriate social media use, but also on how doctors should be using social media.

A medical writer and editor who has worked in the field for 35 years agrees. Pat Rich has led an orientation lecture on appropriate social media use for incoming University of Ottawa medical students for the past six years. He spends a lot of time on Twitter monitoring and interacting on medical health issues and says orientation is the perfect time to delve into the positives and pitfalls of students' online lives.

"When students arrive at medical school, they're often told to get rid of their Facebook and Twitter accounts. I try to bring back balance to the discussion." He describes Twitter as an invaluable resource for young professionals - if they're clear on what they want to get out of it.

"Connect to things you want to read about. Connect to leaders in your specialty around the world - they're often willing to interact via social media and that's hugely valuable." He says the online abuse Gerada 
warns of is rarely an issue when social media is used as a learning tool.

For medical advocates, social media is also an excellent means for putting forth their views to a wide audience. Here Rich cautions that doctors who choose this route must be ready to encounter abuse and pushback. "A more experienced doctor might find it easier to brush that off, but it's much harder to do if you're a student," he says.

Dr. Sara Taylor, a family physician and physician wellness advocate, has also discussed social media with small medical trainee groups, as well as at larger meet- ings such as the Canadian Conference on Physician Health and the Family Medicine Forum. She agrees that, for medical trainees in particular, social media can be uncertain territory and they may feel particularly vulnerable to any ill effects from social media use.

She cites Facebook as a particularly challenging platform to manoeuvre, especially if doctors work at a clinic with a Facebook page. "I have a particular bias against using Facebook for professional use beyond belonging to dedicated groups," she says, citing Physician Side Gigs or Physician Moms as examples.
Like Rich, Taylor asserts that the pros of integrating social media into a medical practice outweigh the cons - as such a key part of our current culture, it's where a lot of conversations are happening. She has three tips: Be transparent and don't misrepresent yourself; don't use social media as a place to vent; and pause before you post anything - before hitting send, always ask yourself whether you would make that statement to a room full of medical professionals, including future employers and program directors.

Sarah Brown, Ottawa, Ont. 